Check for updates

Cite this: RSC Adv., 2018, 8, 25955

Received 2nd June 2018

Accepted 3rd July 2018

DOI: $10.1039 / \mathrm{c} 8 \mathrm{ra04721a}$

rsc.li/rsc-advances

\section{Ultrasensitive enzyme-free fluorescent detection of VEGF 165 based on target-triggered hybridization chain reaction amplification $\dagger$}

\author{
Qingzhen Zhou, $\$^{\mathrm{a}}$ Hongxia Yan, $\dot{t}^{\mathrm{b}}$ Fengying Ran, ${ }^{a}$ Jianjun Cao, ${ }^{a}$ Long Chen, ${ }^{a}$ \\ Bing Shang, ${ }^{a}$ Hao Chen, ${ }^{a}$ Jian Wei ${ }^{\star a}$ and Qinhua Chen (DD *a
}

\begin{abstract}
Sensitive detection of vascular endothelial growth factor $\left(\mathrm{VEGF}_{165}\right)$ is important for early cancer disease diagnosis in the clinic. A sensitive fluorescent sensing platform for VEGF 165 detection is developed in this work. It is based on a target-triggered hybridization chain reaction (HCR) and graphene oxide (GO) selective fluorescence quenching. In this assay, in the presence of the $\mathrm{VEGF}_{165}$, the hairpin structure of $\mathrm{Hp}$ opens up and the initiation sequence will be exposed to Hp1 to open its hairpin structure. Then the opened $\mathrm{Hp} 1$ hybridizes with $\mathrm{Hp} 2$ to expose the complementary sequence of Hp1 which hybridizes with Hp1 again by HCR. Thus HCR would be initiated, generating super-long dsDNA. After the HCR, the double strands of the HCR product cannot be adsorbed on the GO surface. As a result, the HCR product gives a strong fluorescence signal which is dependent on the concentration of VEGF $F_{165}$. By using $V_{E G F} F_{165}$ as a model analyte, the assay provides a highly sensitive fluorescence detection method for $\mathrm{VEGF}_{165}$ with a detection limit down to $20 \mathrm{pg} \mathrm{mL}^{-1}$. The proposed aptasensing strategy based on target-triggered HCR amplification can thus be realized. It was successfully applied to the determination of $\mathrm{VEGF}_{165}$ in spiked human serum, urine and saliva. Therefore, it can easily have wide applications in the diagnosis of vital diseases.
\end{abstract}

\section{Introduction}

Cancer has become one of the greatest causes of human death worldwide, ${ }^{1}$ and so early diagnosis of cancer is particularly important in reducing cancer mortality. Nowadays, techniques for the clinical diagnosis of cancer depend primarily on the biopsy of diseased cells or tissues and imaging equipment. ${ }^{2}$ However, these methods are limited by their low sensitivity in the diagnosis of cancer at an early stage. Biomarkers, including cells, nucleic acids, and proteins, could be used as indicators for the evaluation of either normal or pathological processes. ${ }^{3,4}$ The vascular endothelial growth factor $\left(\mathrm{VEGF}_{165}\right)$ is a significant signal protein stimulating vasculogenesis and is secreted by both endothelial and tumor cells. ${ }^{5}$ It plays a central role in the regulation of the angiogenesis process. ${ }^{6}$ However, VEGF $_{165}$ is usually over-expressed in variants of breast and lung cancer and may be a possible predictor of cancers. ${ }^{7-11}$ VEGF $_{165}$ may be an important biomarker for early cancer disease diagnosis in clinical settings. ${ }^{12,13}$ Thus, the rapid, selective and sensitive

${ }^{a}$ Affiliated Dongfeng Hospital, Hubei University of Medicine, Hubei, Shiyan, 442008, China.E-mail: cqh77@163.com; Tel: +860719-8272283

${ }^{b}$ Department of Radiotherapy, Hubei Cancer Hospital, 116 South Zuodaoquan Road, Wuhan, 430074, China

$\dagger$ Electronic supplementary information (ESI) available. See DOI: 10.1039/c8ra04721a

\$ Qingzhen Zhou and Hongxia Yan are all the first authors. detection of $\mathrm{VEGF}_{165}$ is important for disease diagnosis and subsequent therapy monitoring.

To date, various VEGF $_{165}$ detection techniques have been reported, including ELISA assays, ${ }^{\mathbf{1 4 - 1 6}}$ immunohistochemistry, optical methods, ${ }^{17}$ and radioimmunoassay. However, these methods are mainly time-consuming, labor-intensive, and complex and involve expensive reagents. Several novel biosensor methods have also been reported for the detection of VEGF $_{165}$, such as the use of aptamers. Aptamers are short oligonucleotide sequences (ssDNA or RNA), and could be screened in vitro and synthesized with the systematic evolution of ligands. ${ }^{18}$ The aptamers have high affinity, specificity, and stability, and simple synthesis, easy labeling and wide applicability compared to traditional molecular recognition systems. ${ }^{19}$ Therefore, they are regarded as an alternative to antibody-based methods. Up to now, a variety of aptamer-based analytical strategies for the detection of VEGF $_{165}$ have been reported, such as differential pulse voltammetry (DPV), ${ }^{20,21}$ electrochemical impedance spectroscopy (EIS), ${ }^{\mathbf{1 1}}$ photoelectrochemical (PEC), ${ }^{22}$ electrochemiluminescence (ECL) ${ }^{23}$ chemiluminescence (CL), ${ }^{24,25}$ and time-resolved fluorescence (TR-FL). ${ }^{26}$ These methods have good specificity and stability compared to traditional approaches. ${ }^{27,28}$ However, they lack a signal amplification strategy, resulting in low sensitivity, a narrow detection range and high error rate. For these reasons, a highly sensitive and selective method with a wide quantitative dynamic range is essential for $\mathrm{VEGF}_{165}$ detection. 
Recently, aptamer-based amplification assays, such as the rolling chain reaction (RCA), ${ }^{29}$ polymerase chain reaction assay (PCR) ${ }^{30}$ ligase chain reaction (LCR), ${ }^{31}$ and nanoparticle-assisted amplification $^{32}$ have aroused more attention due to their high sensitivity and wide quantitative dynamic range. However, these methods are highly enzyme-dependent, costly and sensitive to reaction conditions. In addition, the reaction conditions, such as the $\mathrm{pH}$, temperature and buffer media, must be precisely controlled in order to guarantee the activity of the enzymes. ${ }^{33}$

As far as we know, the hybridization chain reaction (HCR), one of the most attractive enzyme-free amplification methods, shows great potential in nucleic acid detection. ${ }^{34,35}$ In an HCR system, two DNA hairpin probes $\mathrm{Hp} 1$ and $\mathrm{Hp} 2$ can coexist stably in solution unless triggered by a primer sequence. ${ }^{36,37}$ When the target was introduced, the hairpin probes changed, initiating a cascade of hybridization events. ${ }^{38}$ Therefore, HCR has been regarded as an ideal choice in DNA-based signal amplification for protein detection. ${ }^{39}$ Graphene oxide (GO) has gained more and more attention due to its large surface area, good water dispersibility and biocompatibility. ${ }^{\mathbf{4 0 , 4 1}}$ Moreover, GO can strongly adsorb single-stranded nucleic acids, but hardly interacts with rigid double-stranded nucleic acids. At the same time, the fluorescence signal of the substances adsorbed by GO can be quenched. Therefore, GO can be used as an ideal nanomaterial in fluorescent biosensors. ${ }^{\mathbf{4 2 , 4 3}}$

Herein, we designed a highly sensitive enzyme-free and rapid aptasensing platform for the detection of $\mathrm{VEGF}_{165}$ based on the HCR. In this assay, in the presence of the $\mathrm{VEGF}_{165}$, the hairpin structure of Hp opens up and the initiation sequence will be exposed to Hp1 to open its hairpin structure by a toeholdmediating strand displacement reaction. Once the hairpin of the first Hp1 opens up, it triggers a cascade reaction, resulting in opening up the hairpin of $\mathrm{Hp} 2$, and consequently $\mathrm{Hp} 1, \mathrm{Hp} 2$, Hp1, Hp2 .... Thus the HCR would be initiated, generating superlong dsDNA. The double strands of the HCR product cannot be adsorbed on the GO surface. ${ }^{44}$ As a result, the HCR product gives a strong fluorescence signal dependent on the concentration of VEGF $_{165}$. More importantly, this simple and rapid amplification strategy can be completed in a short time without expensive enzymes, and provides a novel approach for detection of VEGF $_{165}$. Furthermore, it has been successfully applied to the detection of $\mathrm{VEGF}_{165}$ in various spiked biological samples, with recovery in the range of $99.7-107.2 \%$. Therefore, one may believe that this sensing system possesses great potential for detection of proteins, biological molecules research and clinical diagnosis.

\section{Experimental section}

\subsection{Reagents and materials}

The EpCAM, PSA, BSA and VEGF $_{165}$ were purchased from Cusabio Biotech Co. Ltd. All of the reagents were diluted to the required concentration with working buffer $(20 \mathrm{mM}$ Tris- $\mathrm{HCl}$, $100 \mathrm{mM} \mathrm{NaCl}, \mathrm{pH} 7.4$ ) before use. All of the oligonucleotides used in this work were synthesized and purified using HPLC by Sangon Biotechnology Co. Ltd. (Shanghai, China, https:// www.sangon.com), and their sequences are shown in Table $\mathrm{S} 1 . \dagger \mathrm{GO}$ was purchased from XFNANO Co. Ltd. (Nanjing, China, https://www.xfnano.com). The other reagents employed were of analytical grade and used without further purification. Healthy human serum, urine and saliva were provided by Affiliated Dongfeng Hospital, Hubei University of Medicine, and approved by the Hospital's Ethics Committee. Ultrapure water obtained from a Millipore water purification system (18.2 $\mathrm{M} \Omega \mathrm{cm}$ resistivity, Milli-Q Direct 8) was used in all runs.

\subsection{VEGF $_{165}$ sensing procedure}

Firstly, a mixture of $\mathrm{Hp}$ as well as $\mathrm{Hp} 1$ and $\mathrm{Hp} 2$ strands were separately heated at $90{ }^{\circ} \mathrm{C}$ for $5 \mathrm{~min}$, respectively, followed by slow cooling down to room temperature. Next, different concentrations of $\mathrm{VEGF}_{165}$ were incubated with the Hp solution in working buffer (20 mM Tris- $\mathrm{HCl}, 100 \mathrm{mM} \mathrm{NaCl}, \mathrm{pH} 7.4$ ) for $30 \mathrm{~min}$ at $37^{\circ} \mathrm{C}$. This was followed by the addition of $\mathrm{Hp} 1$ and $\mathrm{Hp} 2$ and incubation at $37{ }^{\circ} \mathrm{C}$. Finally, the GO was added and incubated with the reaction solution for $5 \mathrm{~min}$. Then the solution was diluted to $1 \mathrm{~mL}$, and the fluorescence intensity of the solution was measured.

\subsection{Fluorescence measurements}

The fluorescence detection of the mixtures was carried out on a Hitachi F-4600 spectrophotometer (Hitachi Co. Ltd., Japan, https:/www.hitachi.co.jp) equipped with a xenon lamp excitation source at room temperature. The excitation was set at $495 \mathrm{~nm}$ and the emission spectra were collected from $510 \mathrm{~nm}$ to $600 \mathrm{~nm}$. The slits of the excitation and emission were both set at $5 \mathrm{~nm}$. In control experiments, the measurement process was the same as the above except for the addition of $\mathrm{VEGF}_{165}$. Unless otherwise noted, each fluorescence measurement was repeated three times, and the standard deviation was plotted as the error bar. To evaluate the effects of potential interfering substances on detection, three different relevant compounds, EpCAM, BSA and PSA at a concentration of $100 \mathrm{ng} \mathrm{mL} \mathrm{m}^{-1}$, were spiked, respectively, and the measurements were performed under the same conditions. A quantitative assay of $\mathrm{VEGF}_{165}$ was realized by using the fluorescence intensity. $F_{1}$ and $F_{0}$ are the fluorescence intensities at $519 \mathrm{~nm}$ in the presence and absence of $\mathrm{VEGF}_{165}$, respectively.

\section{Results and discussion}

\subsection{Principle of design}

In the present study, the principle of enzyme-free fluorescent detection of $\mathrm{VEGF}_{165}$ based on HCR is illustrated in Fig. 1. The detection method was composed of three DNA probes, assistant DNA probe (Hp), hairpin probe 1 (Hp1) and hairpin probe $2(\mathrm{Hp} 2)$, and $\mathrm{VEGF}_{165}$ was adopted as the target protein. Hp sequences include a $\mathrm{VEGF}_{165}$ aptamer (in red), extended-DNA (E-DNA, in blue) which is complementary to part of $\mathrm{Hp} 1$ on the $5^{\prime}$ terminal (in yellow), and connecting 10 successive adenine bases (in italics) between them to reduce any possible steric hindrance effect, without interaction with other DNA sequences or molecules. Among these, the Hp1 on the $5^{\prime}$ terminal was labeled with an FAM, and the Hp2 on the $3^{\prime}$ terminal was labeled with an FAM. In the absence of $\mathrm{VEGF}_{165}$, the HCR process cannot be triggered. This results in the coexistence of $\mathrm{Hp}, \mathrm{Hp} 1$ and $\mathrm{Hp} 2$ in solution, which 


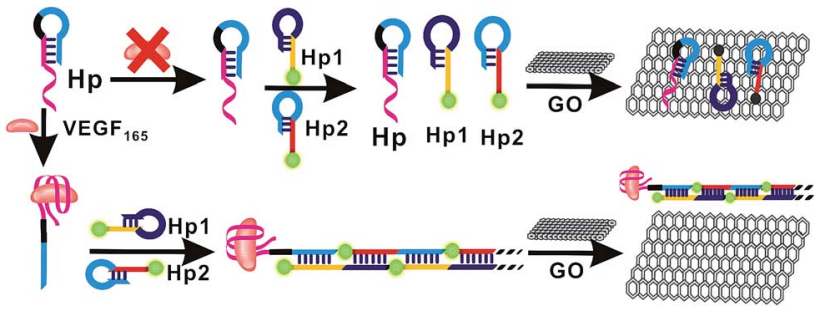

Fig. 1 Schematic illustration of the fluorescence assay detection of VEGF $_{165}$ based on target-triggered hybridization chain reaction amplification.

can be adsorbed on the GO surface through their sticky ends and loops, resulting in a very weak fluorescence signal. In the presence of $\mathrm{VEGF}_{165}$, the aptamer sequences in Hp could be recognized by $\mathrm{VEGF}_{165}$ to form a $\mathrm{VEGF}_{165}$-aptamer complex, and then the E-DNA (in blue) originally caged in Hp was liberated. The liberated E-DNA hybridizes with the sticky end of Hp1 (the yellow region) and opens the hairpin DNA through the toehold-aided strand displacement reaction. The newly released sticky sequence of Hp1 (the dark blue region) further hybridizes with the sticky end of $\mathrm{Hp} 2$ (the red region), to open the Hp2 hairpin DNA and expose a new sticky end on Hp2 (the blue region) being the same as E-DNA. The continuous strand displacement reactions generate a long chain of $\mathrm{Hp}$ leading to complexes of Hp1 and Hp2. Thus the HCR would be initiated, generating super-long dsDNA. After the HCR reaction, the GO was added into the solutions, and the free Hp1 and Hp2 were closely adsorbed onto the GO surface via $\pi-\pi$ stacking. However, the HCR product cannot be adsorbed on the GO surface, ${ }^{45,46}$ realizing a strong fluorescence signal.

\subsection{Feasibility analysis of fluorescence $\mathrm{VEGF}_{165}$ detection}

To further verify the feasibility of the HCR/GO fluorescence signal amplification strategy, Fig. 2 shows the fluorescence emission spectra under different conditions. The fluorescence signal produced by a mixed solution of $\mathrm{Hp} 1$ and $\mathrm{Hp} 2$ (curve a) is

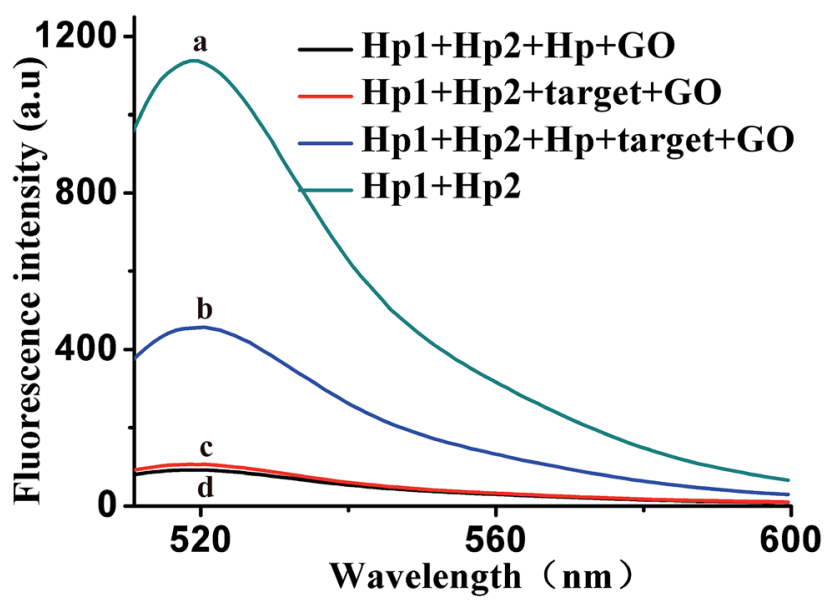

Fig. 2 The typical fluorescent emission spectra of different mixtures, biosensing platform from curve a to d: (a) Hp1 + Hp2; (b) Hp1 + Hp2 + $\mathrm{Hp}+$ target + GO; (c) Hp1 + Hp2 + target + GO; (d) Hp1 + Hp2 + Hp + $\mathrm{GO}$. The concentrations of $\mathrm{Hp1}, \mathrm{Hp} 2, \mathrm{Hp}, \mathrm{GO}$ and target $\left(\mathrm{VEGF}_{165}\right)$ were $30 \mathrm{nM}, 30 \mathrm{nM}, 5 \mathrm{nM}, 20 \mu \mathrm{g} \mathrm{mL}^{-1}$ and $10 \mathrm{ng} \mathrm{mL}^{-1}$, respectively. relatively strong in the absence of GO. After adding the target (curve c) or Hp (curve d), the fluorescence intensity was relatively weak in the presence of GO. The reason may be that, when target and Hp were added alone, the HCR reaction could not occur, giving the results that appear in the graph. However, when $10 \mathrm{ng}$ $\mathrm{mL}^{-1}$ of VEGF $\mathrm{V}_{165}$ was added into a mixed solution of Hp1, Hp2 and $\mathrm{Hp}$, a significant enhancement in the fluorescence intensity was observed (curve b) in the presence of GO, indicating that VEGF $_{165}$ was specifically recognized and triggered the HCR events. These results confirm the feasibility of the proposed fluorescence biosensor for $\mathrm{VEGF}_{165}$ detection by our design.

\subsection{Optimization of reaction conditions}

Whether the fluorescence of $\mathrm{Hp} 1$ and $\mathrm{Hp} 2$ could be effectively quenched by GO is vital for the design of the HCR/GO assay, which is firstly explored in this paper. The fluorescence change is related to the concentration of GO shown in Fig. 3A. The maximum $F_{1} / F_{0}$ value was observed when the concentration of $\mathrm{GO}$ was $20 \mu \mathrm{g} \mathrm{mL}^{-1}$. When the concentration of GO is increased, however, the $F_{1} / F_{0}$ value obviously decreases along with the increasing GO concentration. Therefore, $20 \mu \mathrm{g} \mathrm{mL}^{-1}$ is selected as the optimum GO concentration. At the same time, the reaction time of the HCR is another important factor affecting fluorescence intensity. As shown in Fig. 3B, maximum $F_{1} / F_{0}$ values are observed when the reaction time is $40 \mathrm{~min}$; therefore, 40 min of HCR time was selected for the rest of the experiments.

Besides the above conditions, the $\mathrm{pH}$ and HCR reaction temperature may also affect the sensitivity. As shown in Fig. S1A, $\dagger$ it can be seen that the fluorescence intensity initially increased and then decreased with an increase in $\mathrm{pH}$. The maximum $F_{1} / F_{0}$ values are observed when the $\mathrm{pH}$ is 7.4; therefore, the best $\mathrm{pH}$ was $\mathrm{pH}$ 7.4. Fig. $\mathrm{S} 1 \mathrm{~B} \uparrow$ shows that the incubation temperature could obviously affect the sensitivity, and the $F_{1} / F_{0}$ value reached a maximum when the incubation temperature was $37{ }^{\circ} \mathrm{C}$ and then decreased gradually; thus, an HCR reaction temperature of $37^{\circ} \mathrm{C}$ was selected in the experimental design.

To obtain high sensitivity, the concentrations of $\mathrm{Hp} 1, \mathrm{Hp} 2$ and Hp were also investigated. As shown in Fig. S2A, $\dagger$ the concentration of $\mathrm{Hp} 1$ was selected in the following experiments; the $F_{1} / F_{0}$ value reached a maximum when the concentration of Hp1 was $30 \mathrm{nM}$. Therefore, a concentration of $30 \mathrm{nM}$ was selected as the optimal concentration of $\mathrm{Hp} 1$, and the concentration of Hp2 is identical to that of Hp1. As shown in Fig. S2B, $\uparrow$ the concentration of $\mathrm{Hp}$ was selected in the following experiments. The maximum $F_{1} / F_{0}$ value was observed when the concentration of Hp was $5 \mathrm{nM}$; thus, $5 \mathrm{nM}$ was confirmed as the optimum reaction concentration of $\mathrm{Hp}$.

\subsection{Sensitivity and specificity for $\mathrm{VEGF}_{165}$ detection}

In order to make better use of this designed strategy, the sensitivity of the sensor for the detection of $\mathrm{VEGF}_{165}$ was investigated. As shown in Fig. 4A, in an analysis of different quantities of target, we found that the fluorescence intensity was obviously enhanced with an increased quantity of target. Fig. 4B shows that a good linear correlation between the fluorescence intensity and the logarithm of $\mathrm{VEGF}_{165}$ concentration 
A

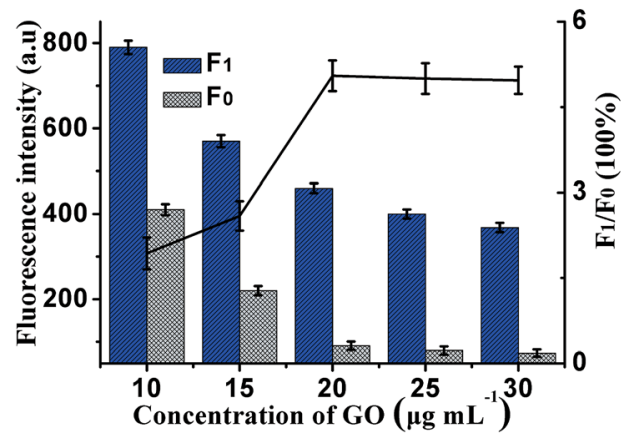

B

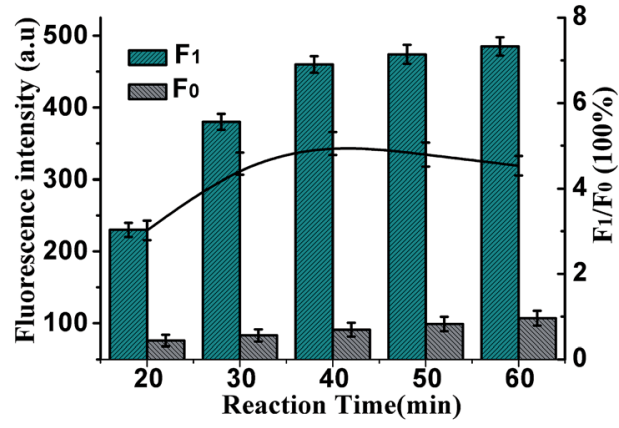

Fig. 3 (A) The effect of GO concentration on the fluorescence response of this method. (B) The fluorescence responses of optimization of HCR time. Light gray columns: control experiments; dark blue and green columns: with $10 \mathrm{ng} \mathrm{mL}^{-1}$ of target; both $\mathrm{Hp} 1$ and $\mathrm{Hp} 2$ are $30 \mathrm{nM}$. The black lines represent the $F_{1} / F_{0}$ at different conditions, where $F_{1}$ and $F_{0}$ are the fluorescence intensities in the presence and absence of $V E G F_{165}$, respectively. Error bars: $S D, n=3$.

A

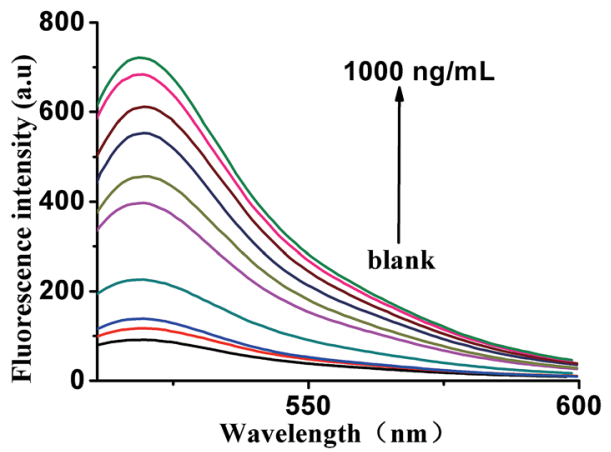

B

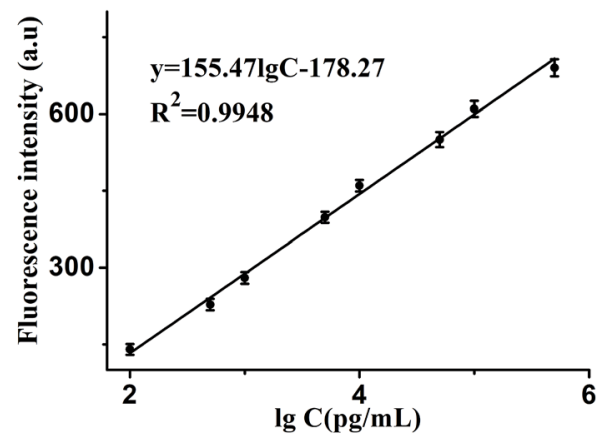

Fig. 4 (A) Fluorescence emission spectra of the biosensor in the presence of VEGF 165 with different concentrations: from bottom to top: 0 to $1000 \mathrm{ng} \mathrm{mL}^{-1}$. (B) The relationship curve of fluorescence intensity as a function of VEGF 165 concentration. It shows the relationship between fluorescence intensity and VEGF 165 concentration, under both experimental conditions: $\mathrm{Hp} 1,30 \mathrm{nM} ; \mathrm{Hp} 2,30 \mathrm{nM} ; \mathrm{Hp}, 5 \mathrm{nM}$; GO, $20 \mu \mathrm{g} \mathrm{mL}{ }^{-1}$ and an emission wavelength of $519 \mathrm{~nm}$. Error bars: SD, $n=3$.

ranging from $0.1 \mathrm{ng} \mathrm{mL}^{-1}$ to $500 \mathrm{ng} \mathrm{mL}^{-1}$ was obtained. The regression equation was expressed as $y=155.471 \log c-178.27$ $\left(R^{2}=0.9948\right)$, where $\log c$ is the logarithm of $\mathrm{VEGF}_{165}$ concentration $\left(\mathrm{pg} \mathrm{mL}^{-1}\right)$. According to the $3 \sigma$ rule, the detection limit

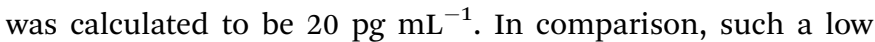
detection limit was comparable to or even higher than those reported in the literature and listed in Table 1.
In addition, the specificity of the sensor was also investigated by adding four kinds of different control proteins, $\mathrm{VEGF}_{165}$, PSA, BSA and EpCAM, as shown in Fig. 5, weak fluorescence was exhibited in the presence of PSA, BSA, or EpCAM, compared with the blank control. However, a significant increase in fluorescence signal was observed in the presence of $\mathrm{VEGF}_{165}$, indicating that this method exhibited good specificity for $\mathrm{VEGF}_{165}$ detection.

Table 1 A comparison of the designed method for the detection of VEGF 165 with other reported biosensors

\begin{tabular}{llll}
\hline Analytical method & Detection limit & Linear range \\
\hline Differential pulse voltammetry & $26.7 \mathrm{pg} \mathrm{mL}^{-1}$ & $0.1-12.2 \mathrm{ng} \mathrm{mL}$ & Ref. \\
Electrochemical impedance spectroscopy & $1.0 \mathrm{pg} \mathrm{mL}^{-1}$ & $10.0-300.0 \mathrm{pg} \mathrm{m}^{-1}$ & $0.003-382 \mathrm{ng} \mathrm{mL}^{-1}$ \\
Photoelectrochemical & $1.1 \mathrm{pg} \mathrm{mL}^{-1}$ & $0.04-764 \mathrm{pg} \mathrm{mL}^{-1}$ & $0.04-4.6 \mathrm{ng} \mathrm{mL}^{-1}$ \\
Electrochemiluminescence & $7.6 \mathrm{pg} \mathrm{mL}^{-1}$ & $1-20 \mathrm{ng} \mathrm{mL}^{-1}$ & 22 \\
Differential pulse voltammetry & $12.2 \mathrm{pg} \mathrm{mL}^{-1}$ & $0.4-611.2 \mathrm{ng} \mathrm{mL}^{-1}$ & 23 \\
Chemiluminescence & $1.0 \mathrm{ng} \mathrm{mL}^{-1}$ & $10-430 \mathrm{ng} \mathrm{mL}^{-1}$ & 21 \\
Time-resolved fluorescence & $3.1 \mathrm{ng} \mathrm{mL}^{-1}$ & $0.1-500 \mathrm{ng} \mathrm{mL}^{-1}$ & 26
\end{tabular}




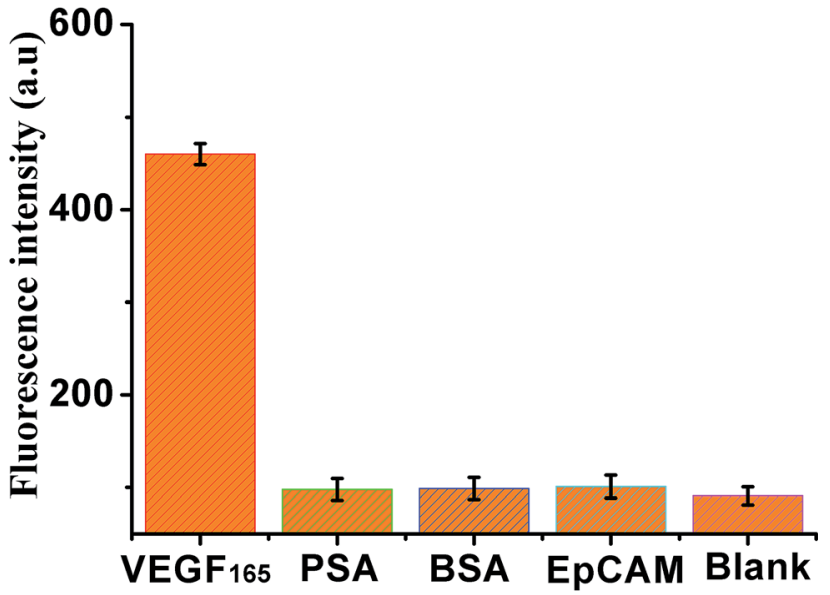

Fig. 5 Fluorescence intensity (at an emission wavelength of $519 \mathrm{~nm}$ ) of the sensor in the presence of VEGF $165\left(10 \mathrm{ng} \mathrm{mL}^{-1}\right)$, PSA (100 $\mathrm{ng}$ $\left.\mathrm{mL}^{-1}\right), \mathrm{BSA}\left(100 \mathrm{ng} \mathrm{mL}^{-1}\right), \operatorname{EpCAM}\left(100 \mathrm{ng} \mathrm{mL}^{-1}\right)$ and black, respectively. Error bars: SD, $n=3$

\subsection{Determination of $\mathrm{VEGF}_{165}$ in real samples}

To assess the application of this proposed design in biological samples (all spiked samples were diluted to 10\%), human serum, urine and saliva, respectively, were employed as a complex matrix. Before the measurements, the samples were prepared by adding VEGF $_{165}$ into blank biological samples including human serum, urine and saliva, respectively. As shown in Fig. 6, the enhancement in fluorescence intensity is observed in various biological samples in the presence of $10 \mathrm{ng}$ $\mathrm{mL}^{-1} \mathrm{VEGF}_{165}$, compared with unspiked biological samples. The results indicate that use of the proposed sensor to analyze VEGF $_{165}$ in biological samples was feasible. The recoveries of VEGF $_{165}$ spiked to different matrices are listed in Table 2. The recoveries for the spiked $\mathrm{VEGF}_{165}$ in human serum, saliva and urine were in the range of $107.2-99.7 \%$, and the RSD (\%) were $10.8 \%, 10.3 \%, 9.9 \%$ at $10 \mathrm{ng} \mathrm{mL} \mathrm{mL}^{-1}$ of $\mathrm{VEGF}_{165}$, respectively. This indicates an acceptable precision and reproducibility of the present approach for detecting $\operatorname{VEGF}_{165}$ in real samples $(n=3)$.

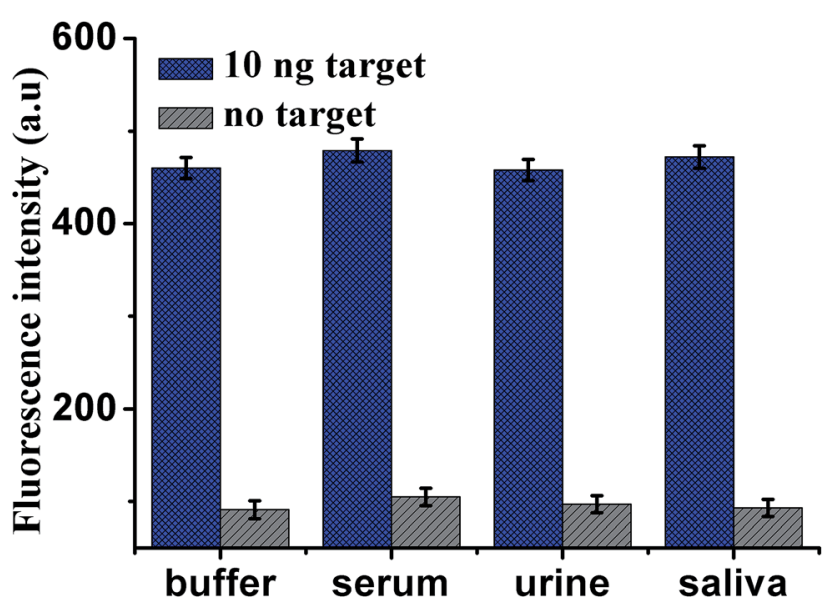

Fig. 6 Fluorescence intensity of the sensor for the detection of VEGF $_{165}$ in buffer and blank biological samples (human serum, urine and saliva, respectively). Error bars: SD, $n=3$.
Table 2 Recoveries of $\mathrm{VEGF}_{165}$ spiked to different matrices

\begin{tabular}{|c|c|c|c|c|}
\hline Matrix & $\begin{array}{l}\text { VEGF }_{165} \text { added/ } \\
\text { ng }\end{array}$ & $\begin{array}{l}\mathrm{VEGF}_{165} \text { found/ } \\
\mathrm{ng}\end{array}$ & $\begin{array}{l}\text { Recovery } \\
(100 \%)\end{array}$ & RSD (\%) \\
\hline Buffer & 10.0 & $9.98^{a}$ & 99.8 & 9.6 \\
\hline Serum & 10.0 & $10.72^{a}$ & 107.2 & 10.8 \\
\hline Saliva & 10.0 & $10.26^{a}$ & 102.6 & 10.3 \\
\hline Urine & 10.0 & $9.97^{a}$ & 99.7 & 9.9 \\
\hline
\end{tabular}

\section{Conclusions}

In conclusion, a novel and enzyme-free highly sensitive assay of fluorescent signal amplification was successfully developed for detecting VEGF $_{165}$. In this design, because of the specific recognition of aptamer for $\mathrm{VEGF}_{165}$ and signal amplification by HCR, selectivity and sensitivity for VEGF $_{165}$ were achieved. Besides, the HCR reaction has strong environmental adaptability due to there being no participation of enzymes. Under optimal conditions, the detection limit was as low as $20 \mathrm{pg} \mathrm{mL}^{-1}$, and a linearity ranging from $0.1 \mathrm{ng} \mathrm{mL}^{-1}$ to $500 \mathrm{ng} \mathrm{mL}^{-1}$ was obtained. More importantly, this biosensor was successfully applied for the detection of VEGF $_{165}$ in various spiked biological samples, and provides a novel approach for the detection of $\mathrm{VEGF}_{165}$ compared to other reported biosensors. One may believe that it could be a potential method for proteomics and clinical analysis.

\section{Conflicts of interest}

There are no conflicts to declare.

\section{Acknowledgements}

These works were supported by the Natural Science Foundation of Hubei Provincial Department of Education (No. D20172101), the School Foundation for Hubei University of Medicine (No. FDFR201614), the Scientific research project of Hubei Provincial Department of Education (No. Q20162113 and B2016137), the Health Department of Hubei Province (No. QJX2010-51), the Science and Technology Key Program of Shiyan (No. 14Y54, 17 Y56 and 15Y49), and the Key Discipline Project of Hubei University of Medicine.

\section{References}

1 W. Chen, R. Zheng, P. D. Baade, S. Zhang, H. Zeng, F. Bray, A. Jemal, X. Q. Yu and J. He, Ca-Cancer J. Clin., 2016, 66, 115132.

2 A. B. Chinen, C. M. Guan, J. R. Ferrer, S. N. Barnaby, T. J. Merkel and C. A. Mirkin, Chem. Rev., 2015, 115, 10530-10574.

3 O. Golubnitschaja and J. Flammer, Surv. Ophthalmol., 2007, 52(suppl. 2), S155-S161.

4 N. V. T. U. Hohensteinblaul, S. Kunst, N. Pfeiffer and F. H. Grus, Eye, 2017, 31, 225-231. 
5 E. Itakura, H. Yamamoto, Y. Oda and M. Tsuneyoshi, J. Surg. Oncol., 2008, 97, 74-81.

6 D. R. Senger, S. J. Galli, A. M. Dvorak, C. A. Perruzzi, V. S. Harvey and H. F. Dvorak, Science, 1983, 219, 983-985.

7 M. Enjoji, M. Nakamuta, K. Yamaguchi, S. Ohta, K. Kotoh, M. Fukushima, M. Kuniyoshi, T. Yamada, M. Tanaka and H. Nawata, World J. Gastroenterol., 2005, 11, 1167-1171.

8 A. M. Granato, O. Nanni, F. Falcini, S. Folli, G. Mosconi, F. D. Paola, L. Medri, D. Amadori and A. Volpi, Breast Cancer Res., 2004, 6, R38-R45.

9 E. Dalaveris, T. Kerenidi, A. Katsabeki-Katsafli, T. Kiropoulos, K. Tanou, K. I. Gourgoulianis and K. Kostikas, Lung Cancer, 2009, 64, 219-225.

10 R. M. Loureiro and P. A. D'Amore, Cytokine Growth Factor Rev., 2005, 16, 77-89.

11 M. A. Tabrizi and M. Shamsipur, Biosens. Bioelectron., 2015, 74, 764-769.

12 M. Skobe, T. Hawighorst, D. G. Jackson, R. Prevo, L. Janes, P. Velasco, L. Riccardi, K. Alitalo, K. Claffey and M. Detmar, Nat. Med., 2001, 7, 192-198.

13 H. S. Lee, K. S. Kim, C. J. Kim, S. K. Hahn and M. H. Jo, Biosens. Bioelectron., 2009, 24, 1801-1805.

14 X. Lin, K. H. Leung, L. Lin, L. Lin, S. Lin, C. H. Leung, D. L. Ma and J. M. Lin, Biosens. Bioelectron., 2016, 79, 41-47.

15 M. Y. Hsu, S. J. Chen, K. H. Chen, Y. C. Hung, H. Y. Tsai and C. M. Cheng, Lab Chip, 2015, 15, 2357-2363.

16 H. Takahashi, Y. Nomura, J. Nishida, Y. Fujino, Y. Yanagi and H. Kawashima, Invest. Ophthalmol. Visual Sci., 2016, 57, 462-466.

17 K. Kopra, M. Syrjanpaa, P. Hanninen and H. Harma, Analyst, 2014, 139, 2016-2023.

18 R. Y. Lai, K. W. Plaxco and A. J. Heeger, Anal. Chem., 2007, 79, 229-233.

19 O. Neumann, D. Zhang, F. Tam, S. Lal, P. Wittung-Stafshede and N. J. Halas, Anal. Chem., 2009, 81, 10002-10006.

20 M. A. Tabrizi, M. Shamsipur, R. Saber and S. Sarkar, Sens. Actuators, B, 2016, 240, 1174-1181.

21 M. Shamsipur, L. Farzin, T. M. Amouzadeh and F. Molaabasi, Biosens. Bioelectron., 2015, 74, 369-375.

22 H. Da, H. Liu, Y. Zheng, R. Yuan and Y. Chai, Biosens. Bioelectron., 2018, 101, 213-218.

23 H. Zhang, M. Li, C. Li, Z. Guo, H. Dong, P. Wu and C. Cai, Biosens. Bioelectron., 2015, 74, 98-103.

24 S. Shan, Z. He, S. Mao, M. Jie, L. Yi and J. M. Lin, Talanta, 2017, 171, 197-203.

25 H. Zhang, L. Peng, M. Li, J. Ma, S. Qi, H. Chen, L. Zhou and X. Chen, Analyst, 2017, 142, 2419-2425.
26 D. Zhu, W. Li, H. M. Wen, S. Yu, Z. Y. Miao, A. Kang and A. Zhang, Biosens. Bioelectron., 2015, 74, 1053-1060.

27 R. A. Potyrailo, A. J. Murray, N. Nagraj, A. D. Pris, J. M. Ashe and M. Todorovic, Angew. Chem., Int. Ed. Engl., 2015, 54, 2174-2178.

28 S. E. Lee, Q. Chen, R. Bhat, S. Petkiewicz, J. M. Smith, V. E. Ferry, A. L. Correia, A. P. Alivisatos and M. J. Bissell, Nano Lett., 2015, 15, 4564-4570.

29 Q. Xue, Z. Wang, L. Wang and J. Wei, Bioconjugate Chem., 2012, 23, 734-739.

30 Y. Yoshida, K. Horii, N. Sakai, H. Masuda, M. Furuichi and I. Waga, Anal. Bioanal. Chem., 2009, 395, 1089-1096.

31 W. S. Chan, B. S. Tang, M. V. Boost, C. Chow and P. H. Leung, Biosens. Bioelectron., 2014, 53, 105-111.

32 W. Song, H. Li, H. Liang, W. Qiang and D. Xu, Anal. Chem., 2014, 86, 2775-2783.

33 X. Chen, N. Briggs, J. R. Mclain and A. D. Ellington, Proc. Natl. Acad. Sci. U. S. A., 2013, 110, 5386-5391.

34 Y. Chang, Y. Chai, S. Xie, Y. Yuan, J. Zhang and R. Yuan, Analyst, 2014, 139, 4264-4269.

35 H. M. Choi, V. A. Beck and N. A. Pierce, ACS Nano, 2014, 8, 4284-4294.

36 Z. Wu, G. Q. Liu, X. L. Yang and J. H. Jiang, J. Am. Chem. Soc., 2015, 137, 6829-6836.

37 H. M. T. Choi, J. Y. Chang, L. A. Trinh, J. E. Padilla, S. E. Fraser and N. A. Pierce, Nat. Biotechnol., 2010, 28, 1208-1212.

38 T. Zhao, H. S. Zhang, H. Tang and J. H. Jiang, Talanta, 2017, 175, 121-126.

39 S. Dai, Q. Xue, J. Zhu, Y. Ding, W. Jiang and L. Wang, Biosens. Bioelectron., 2014, 51, 421-425.

40 K. L. Seng, P. Chen, F. L. Lee, S. Moochhala and L. Bo, Anal. Chem., 2015, 87, 9408-9412.

41 S. C. Ray, S. K. Bhunia, A. Saha and N. R. Jana, Microelectron. Eng., 2015, 146, 48-52.

42 J. Huang, X. Gao, J. Jia, J. K. Kim and Z. Li, Anal. Chem., 2014, 86, 3209-3215.

43 B. Feng, L. Guo, L. Wang, F. Li, J. Lu, J. Gao, C. Fan and Q. Huang, Anal. Chem., 2013, 85, 7732-7737.

44 D. Xiao, Y. Li, L. Liu, B. Wen, Z. Gu, C. Zhang and Y. S. Zhao, Chem. Commun., 2012, 48, 9519-9521.

45 C. Ma, H. Liu, T. Tian, X. Song, J. Yu and M. Yan, Biosens. Bioelectron., 2016, 83, 15-18.

46 S. Guo, F. Yang, Y. Zhang, Y. Ning, Q. Yao and G. J. Zhang, Anal. Methods, 2014, 6, 3598-3603. 